\title{
Isolierung und Quantifizierung nicht-sulfatierter und sulfatierter Gallensäuren im Stuhl
}

\author{
Von N. Breuer, P. Dommes, R. Tandon und H. Goebell \\ Abteilung für Gastroenterologie, Medizinische Klinik und Poliklinik der Universität Essen (GHS)
}

(Eingegangen am 24. November 1983/19. März 1984)

\begin{abstract}
Zusammenfassung: Es wird eine Methode zur qualitativen und quantitativen Bestimmung von nicht-sulfatierten und sulfatierten Gallensäuren im Stuhl beschrieben. Nach Vorreinigung der Gallensäuren können diese durch Chromatographie an DEAP-Sephadex LH20 in freie, konjugierte und sulfatierte Gallensäuren aufgetrennt werden. In diesen Fraktionen kann dann, gegebenenfalls nach Solvolyse und Hydrolyse, mittels Gaschromatographie eine qualitative und quantitative Analyse der einzelnen Gallensäuren erfolgen. Die Validierung der einzelnen Analysenschritte und die Überprüfung des gesamten Analysenganges durch dem Stuhl zugesetzte Gallensäuren als interne Standards wiesen bei guter Reproduzierbarkeit eine gute Trennung nichtsulfatierter und sulfatierter Gallensäuren nach. Die Untersuchung von Stuhlproben bei 15 Referenzpersonen ergab eine durchschnittliche Gallensäure-Ausscheidung von $3,85 \mathrm{mg} / \mathrm{g}$ Trockenstuhl, wobei $10,4 \%$ auf die primären und $89,6 \%$ auf die sekundären Gallensäuren entfielen; $92,9 \%$ der fäkalen Gallensäuren lagen als freie, $2,7 \%$ als konjugierte Gallensäuren vor. Der Anteil der sulfatveresterten Gallensäuren betrug nur 4,4\%.
\end{abstract}

\section{Isolation and quantification of non-sulphated and sulphated bile acids in faeces}

Summary: A method is described for the qualitative and quantitative analysis of non-sulphated and sulphated bile acids in faeces. After extraction and preliminary purification, the faecal bile acids are separated by liquidgel-chromatography (DEAP-Sephadex LH20) into free, conjugated and sulphated bile acids; these are quantitated separately (after solvolysis and hydrolysis), and the individual bile acids are analysed by gas-liquidchromatography. The validation both of the individual analysis steps and the overall procedure by adding bile acid standards to the faecal homogenates showed a good reproducibility and a reliable separation of non-sulphated and sulphated bile acids. Using the described method, the excretion of total faecal bile acids in 15 control subjects was $3.85 \mathrm{mg} / \mathrm{g}$ dry stool, consisting of $10.4 \%$ primary bile acids and $89.6 \%$ secondary bile acids. $92.9 \%$ of faecal bile acids were in the free form, only $2.7 \%$ in the conjugated form, and $4.4 \%$ as sulphated bile acids.

\section{Einfüihrung}

Die in der Leber aus Cholesterin synthetisierten (primären) Galleñäuren Chenodesoxycholsäure und Cholsäure werden fast ausschließlich als Glycinoder Taurin-Konjugate in den Dünndarm sezerniert, von wo sie zu 95 bis $98 \%$ rückresorbiert werden (1).

Durch bakterielle Enzyme werden die Gallensäuren, zum geringen Teil im Ileum, zum größten Teil im Colon, zu freien Gallensäuren dekonjugiert und zu den sekundären Gallensäuren Lithocholsäure und
Desoxycholsäure dehydroxyliert. Diese werden zum Teil ebenfalls rückresorbiert. Die dem enterohepatischen Kreislauf entgehenden Gallensäuren werden zum Teil weiter bakteriell verändert und im Stuhl ausgeschieden.

Mit der hepatischen Sulfatierung, die für viele Substanzen einen allgemeinen Mechanismus der Biotransformation darstellt, wurde 1967 (2) ein zusätzlicher Stoffwechselweg der Gallensäuren beschrieben. Diesem kommt eine besondere Bedeutung bei 
Cholestase-Syndromen zu (3-6); hierbei machen die Sulfatester, die neben einer größeren Wasserlöslichkeit auch eine geringere Toxizität für die Leber $(7,8)$ und den Darm (9) besitzen, den größten Teil der renal ausgeschiedenen Gallensäuren aus (10). Wie in der Niere, werden sulfatierte Gallensäuren auch im Dünndarm weniger gut als nicht-sulfatierte Gallensäuren rückresorbiert (11-13), so daß ihre Ausscheidung in den Faeces ansteigt $(12,14)$. Angaben darüber, welchen mengenmäßigen Anteil sulfatierte Gallensäuren normalerweise an der fäkalen Gallensäureausscheidung haben, differieren aus methodischen Gründen erheblich (15-18). Der Grad der Desulfatierung durch Bakterien spielt dabei ebenfalls eine Rolle (12).

Wir beschreiben deshalb eine Methode zur Bestimmung der fäkalen Gallensäuren, mit der die sulfatierten Gallensäuren von den nicht-sulfatierten säulenchromatographisch getrennt und separat quantifiziert werden. 'Darüber hinaus werden die an $15 \mathrm{Re}-$ ferenzpersonen ermittelten Ergebnisse bezüglich des Gallensäuremusters im Stuhl mitgeteilt.

\section{Materialien und Methoden}

\section{Referenzkollektiv}

Bei 15 Patienten unserer Klinik, bei denen sich auf Grund der klinischen Untersuchung und der routinemäßig durchgeführten Laboruntersuchungen kein Anhalt für eine gastrointestinale Erkrankung ergab, bestimmten wir die Gallensäureausscheidung im Stuhl (mg/g Trockenstuhl). Bezüglich der Ernährungsgewohnheiten waren wesentliche Unterschiede nicht offensichtlich. Spezielle Diäten wurden nicht befolgt.

\section{Reagentien}

Alle Reagentien und Lösungsmittel waren, soweit nicht anders angegeben, von E. Merck, Darmstadt. Gallensäurestandards wurden von Sigma, München, Paesel, Frankfurt und Pl-Biochemicals, St. Goar, bezogen. $\left[{ }^{14} \mathrm{COOH}-\right]$ Lithocholsäure (spezif. Aktivität $2,183 \mathrm{TBq} / \mathrm{mol} \cong 59 \mathrm{~m} \mathrm{Ci} / \mathrm{mmol}$ ) von Amersham Buchler $\mathrm{GmbH}$ \& Co KG, Braunschweig; sämtliche Gallensäuren wiesen dünnschichtchromatographisch einen Reinheitsgrad $>99 \%$ auf. XAD2-Harz und Amberlyst-15-Ionenaustauscher, beide analytical grade, waren von Serva, Heidelberg. DEAP-Sephadex LH20 wurde unter der Bezeichnung Lipidex ${ }^{\text {TM}}$-DEAP von Packard Instruments, Frankfurt, bezogen. Sep $\mathrm{Pak}^{\mathrm{TM}}-\mathrm{C}_{18}-\mathrm{Kartuschen}$ waren von Waters Ass., Königsstein/Ts. Cholylglycin-Hydrolase aus $C$. perfringens (welchii) (100 U/mg Protein) lieferte Sigma, München. Als Szintillator wurde Unisolve I von Zinsser Analytic $\mathrm{GmbH}$, Frankfurt/M, benutzt.

\section{Geräte}

Zur Trocknung der Stuhlproben wurde eine Gefriertrocknungsanlage Lo5 (-60) der Firma WKF, Brandau/Darmstadt, benutzt. Zur gaschromatographischen Analyse diente ein Packard Becker Gaschromatograph Modell 419 von Packard Instruments, Frankfurt. Die Auswertung der Gaschromatogramme erfolgte mit einem SP 4100 Computing Integrator der Firma Spectra Physics, Darmstadt. Zur Messung der ${ }^{14} \mathrm{C}$-Aktivität wurde ein Flüssigkeitsszintillationszähler Kontron MR 300 DPM von Kontron Analytik GmbH, Eching/München, benutzt.

\section{Methoden}

\section{$X A D$-2-Chromalographie}

Amberlite XAD-2 wird in eine Glassäule (innerer Durchmesser $0,6 \mathrm{~cm}$, Länge $30 \mathrm{~cm}$ ) zu einem Bettvolumen von $8,5 \mathrm{ml}$ gefüllt. Die Säule wird mit Wasser ( $\mathrm{pH} 7,0)$ äquilibriert.

\section{Amberlyst-15-Chromatographie}

Amberlyst-15 wird in eine Glassäule (innerer Durchmesser $0,6 \mathrm{~cm}$, Länge $30 \mathrm{~cm}$ ) zu einem Bettvolumen von $8,5 \mathrm{ml}$ gefüllt. Der Ionenaustauscher wird durch Spülen mit $\mathrm{HCl}(2 \mathrm{~mol} / \mathrm{l})$ in 700 $\mathrm{ml} / /$ Ethanol in die protonierte Form überführt. Anschließend wird die Säule mit $720 \mathrm{ml} / \mathrm{l}$ Ethanol gewaschèn.

\section{DEAP-Sephadex LH20-Chromatographie}

DEAP-Sephadex LH20 wird entsprechend der Vorschrift von Alme et al. (19) vorbereitet und hat einen Amingehalt von 1,2 $\mathrm{mmol} / \mathrm{g}$ Trockensubstanz. Trockenes Gel $(0,5 \mathrm{~g})$ wird in $720 \mathrm{ml} / \mathrm{l}$ Ethanol $(\mathrm{pH} 7,0)$ aufgeschlemmt und in eine Glassäule (innerer Durchmesser $0,5 \mathrm{~cm}$ ) zu einer Höhe von $11 \mathrm{~cm}$ gefüllt (Gelbettvolumen etwa $2,2 \mathrm{ml}$ ).

Die Probe, gelöst in insgesamt $4 \mathrm{ml} 720 \mathrm{ml} /$ Ethanol (pH 7,0) wird aufgetragen und mit Druckluft $(25 \mathrm{kPa} \bumpeq 0,25 \mathrm{~atm})$ in das Gel gedrụ̈ckt. Die Auftrennung der freien, konjugierten und sulfatierten Gallensäuren in drei entsprechende Fraktionen erfolgt im wesentlichen nach der Methode von Alme et al. (19). Durch Modifikation der Elutionspuffer und -volumina werden jedoch Glycin- und Taurin-konjugierte Gallensäuren in einer Fraktion vereinigt. Das Elutionsschema ist in Tabelle 1 wiedergegeben. Die Elutionspuffer werden ebenfalls mit einem Uberdruck von 25 $\mathrm{kPa} \cong 0,25 \mathrm{~atm}$ durch das Gel gedrückt, womit eine konstante Flußgeschwindigkeit von $22 \mathrm{ml} / \mathrm{h}$ erreicht wird.

Tab. 1. Elutionsschema der DEAP-Sephadex LH20 Säule.

\begin{tabular}{|c|c|c|c|c|}
\hline \multirow[t]{3}{*}{$\begin{array}{l}\text { Frak- } \\
\text { tion }\end{array}$} & \multirow[t]{3}{*}{ Eluierte Substanzen } & \multicolumn{3}{|c|}{$\begin{array}{l}\text { Elutionsmittel: } 720 \mathrm{ml} / \mathrm{l} \\
\text { Ethanol }\end{array}$} \\
\hline & & $\begin{array}{l}\text { Vo- } \\
\text { lumen }\end{array}$ & $\begin{array}{l}\text { Acetat- } \\
\text { Konzen- } \\
\text { tration }\end{array}$ & $\mathrm{pH}^{*}$ \\
\hline & & (ml) & $(\mathrm{mol} / \mathrm{l})$ & \\
\hline 1 & Neutrale Steroide & 10 & 0,0 & 7,0 \\
\hline 2 & Freie Gallensäuren & 12 & 0,10 & 3,9 \\
\hline 3 & Konjugierte Gallensäuren & 10 & 0,15 & 6,6 \\
\hline 4 & Sulfatierte Gallensäuren & 12 & 0,30 & 9,6 \\
\hline
\end{tabular}

* mit konz. $\mathrm{NH}_{3}$-Lösung eingestellt.

\section{Hydrolyse}

Die enzymatische Hydrolyse der mit Glycin oder Taurin konjugierten Gallensäuren erfolgt nach der Methode von Roseleur \& van Gent (20). Die in $25 \mathrm{mmol} / \mathrm{A}$ Natriumacetat gelöste Probe wird mit 200 U Cholylglycin-Hydrolase versetzt. Das Reaktionsgemisch (etwa $6,5 \mathrm{ml}$ ) wird $16 \mathrm{~h}$ bei $37^{\circ} \mathrm{C}$ inkubiert und danach durch eine Sep Pak ${ }^{\mathrm{TM}}-\mathrm{C}_{18}-\mathrm{Kartusche}$ gedrückt, die anschließend mit $10 \mathrm{ml}$ Wasser (pH 7,0) gespült wird. Die Gallensäuren werden mit 1,0 ml Methanol eluiert, wobei die ersten 10 Tropfen (bis zum Austritt des Methanols aus der, Kartusche) verworfen werden. 


\section{Solvolyse}

Dic Spaltung der Sulfatester der Gallensäuren wird in Anlehnung an die Methode von van Berge-Henegouwen (21) durchgeführt. Die etherische Salzsäurelösung wird durch sorgfältige Destillation einer Mischung von $60 \mathrm{ml}$ Ether und $40 \mathrm{ml}$ konz. Salzsäure über eine Vigreux-Kolonne $(30 \mathrm{~cm})$ hergestellt. Die ersten $10 \mathrm{ml}$ des Destillats werden verworfen. Dann werden $30 \mathrm{ml}$ des bei etwa $35^{\circ} \mathrm{C}$ übergehenden Destillats gesammelt und bei $-20^{\circ} \mathrm{C}$ aufbewahrt. Die Probe, die zunächst in $0,1 \mathrm{ml}$ Methanol gelöst wird, um die Entstehung von Artefakten bei der Solvolyse zu vermeiden, wird mit $1,0 \mathrm{ml}$ etherischer Salzsäure versetzt und $24 \mathrm{~h}$ bei Raumtemperatur umgesetzt.

\section{Gaschromatographie}

Die freien Gallensäuren werden, gegebenenfalls nach Hydrolyse und Solvolyse, mit Diazomethan in Ether/Methanol (1 ml, 1:10) verestert und anschließend mit Trifluoressigsäureanhydrid $30 \mathrm{~min}$ bei $37^{\circ} \mathrm{C} \mathrm{zu}$ den entsprechenden Trifluoressigsäureestern umgesetzt. Zur Korrektur der Einspritzvolumina wurde jede Probe vor der Derivatisierung mit 3a-Hydroxy-6-oxo-5 $\alpha$-cholansäure (Endkonzentration in der Probe $1 \mathrm{mmol} / 1$ ) versetzt. Bedingungen: $1 \%$ QF1 auf Gaschrom Q (100/120 mesh). Säulenlänge $180 \mathrm{~cm}$, innerer Durchmesser $2 \mathrm{~mm}$. Trägergas: Helium, $45 \mathrm{ml} /$ min. Säulentemperatur $230^{\circ} \mathrm{C}$ isotherm; Flammen-IonisationsDetektor $260^{\circ} \mathrm{C}$, Injection port $250^{\circ} \mathrm{C}$. Die Auswertung der Chromatogramme erfolgt über eine Integration der Peakflächen, wobei mittels einer zuvor erstellten ,Multilevel-Calibration-Kurve" die unterschiedliche Detektorempfindlichkeit für die individuellen Gallensäuren, auch als Funktion ihrer Konzentration, in Rechnung gesetzt wurden.

\section{Beschreibung des Analysenganges}

Der gesamte Analysengang zur Bestimmung fäkaler Gallensäuren ist zur besseren Ubersicht in Abbildung 1 dargestellt.

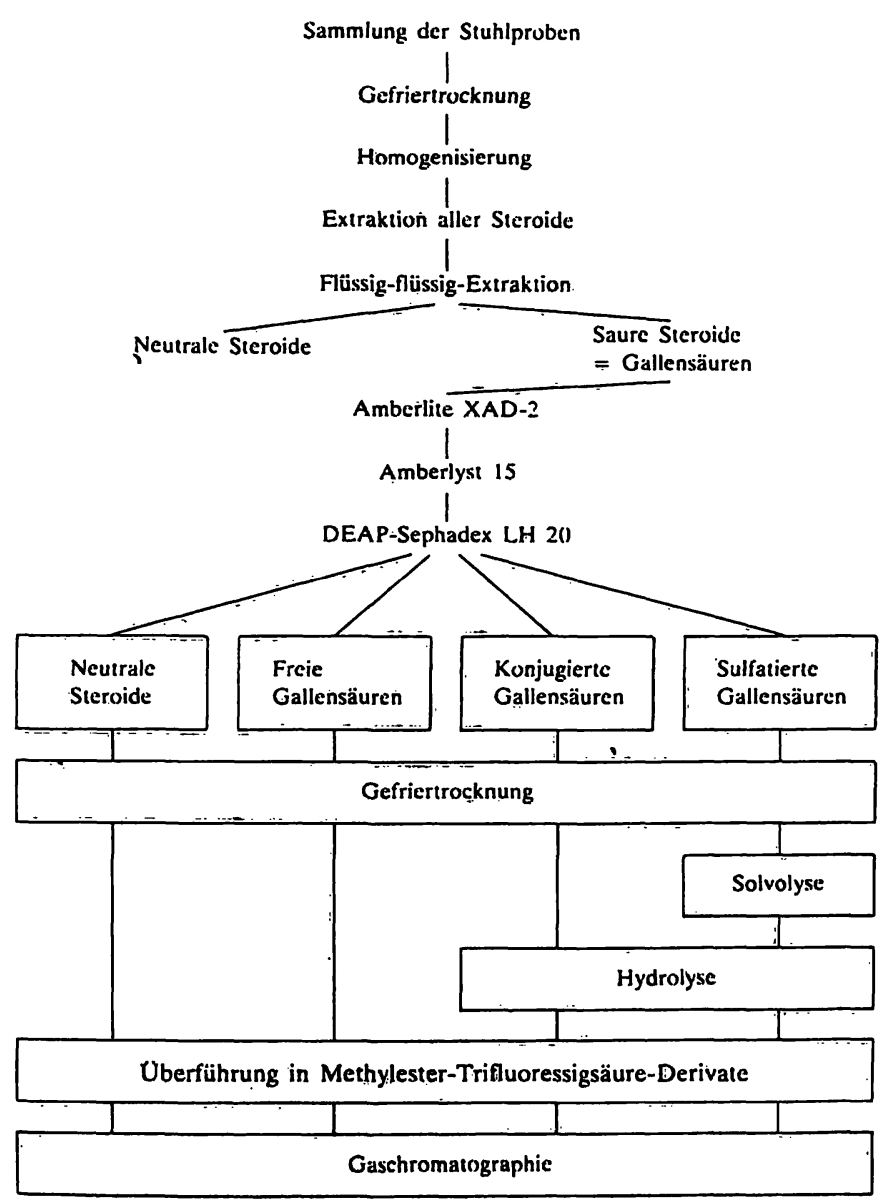

Abb. 1. Flußdiagramm zur Analyse von fäkalen Gallensäuren.
Die Sammlung der Stuhlproben erfolgt über 3 Tage, wobei jeder einzelne Stuhl unmittelbar nach Defäkation bei $-30^{\circ} \mathrm{C}$ eingefroren wird, um einen bakteriellen Abbau der Gallensäuren zu vermeiden. Die anschließende Gefriertrocknung erfolgt bis zur Gewichtskonstanz der Probe. Nach der mechanischen Homogenisierung des gefriergetrockneten Materials werden alle Trockenstühle entsprechend ihren Trockengewichten vereinigt. Hiervon wird $1 \mathrm{~g}$ zur Extraktion mit $100 \mathrm{ml} 900 \mathrm{ml} / \mathrm{E}$ Ethanol am Rückfluß $(2 \mathrm{~h})$ verwendet. Nach Zentrifugation $(15 \mathrm{~min}, 15000 \mathrm{~g}$ ) wird das Sediment in $100 \mathrm{ml} 800 \mathrm{ml} / \mathrm{l}$ Ethanol aufgenommen und weitere $2 \mathrm{~h}$ extrahiert. Nach erneuter Zentrifugation wird das Sediment abschließend mit $100 \mathrm{ml}$ Chloroform/Methanol (Volumina, 1 + 1) am Rückfluß extrahiert. Der bei der anschließenden Zentrifugation anfallende Uberstand wird mit den Uberständen der vorausgegangenen Zentrifugationen vereinigt und zur Trockne eingeengt.

Zur Flüssig-Flüssig-Extraktion wird der Rückstand in $100 \mathrm{ml} 72.0$ $\mathrm{ml} / \mathrm{l}$ Ethanol aufgenommen und $\mathrm{mit} 1 \mathrm{ml}$ konz. Ammoniaklösung alkalisch gemacht (pH 11). Die Lösung wird zur Extraktion der neutralen Steroide dreimal mit je $100 \mathrm{ml} n$-Hexan ausgeschüttelt. $1 / 10$ der ethanolischen Phase wird zur Trockne eingeengt (der Rest wird für weitere Bestimmungen eingefroren). Der Rückstand wird mit insgesamt $10 \mathrm{ml} 88 \mathrm{mmol} / \mathrm{l}$ Ammoniumcarbonatlösung aufgenommen und auf das $X A D-2$-Harz gegeben. Die Säule wird mit $100 \mathrm{ml}$ Wasser (pH 7,0) gespült. Die an das Harz adsorbierten Gallensäuren werden mit $50 \mathrm{ml} 720 \mathrm{ml} / /$ Ethanol (pH 7,0) eluiert. Dieses Eluat wird direkt auf die Amberlyst-15lonenaustauschersäule gegeben; das Harz wird anschließend mit $50 \mathrm{ml} 720 \mathrm{ml} / /$ Ethanol (pH 7,0) gewaschen. Durchlauf und Eluat der Säule (insgesamt $100 \mathrm{ml}$ ) werden zur Trockne abgezogen. Der Rückstand wird mit insgesamt $4 \mathrm{ml} 720 \mathrm{ml} / 1$ Ethanol (pH 7,0) aufgenommen und auf das DEAP-Sephadex LH20-Gel gegeben. Die Säule wird dann, wie oben angegeben, eluiert; die 4 Fraktionen werden zur Entfernung des Ammoniumacetats lyophilisiert. Fraktion 4 (mit sulfatierten Gallensäuren) wird zunächst solvolysiert, dann wie Fraktion 3 (mit konjugierten Gallensäuren) hydrolysiert. Nach Vorliegen der freien Gallensäuren können diese Fraktionen zusammen mit Fraktion 1 (mit neutralen Steroiden) und Fraktion 2 (mit freien Gallensäuren) nach Zugabe des internen Standards ( $3 \alpha$-Hydroxy-6-oxo-5 $\alpha$-cholansäure, $1 \mathrm{mmol} / \mathrm{l}$ ), wie im Abschnitt Gaschromatographie beschrieben, in die Methylester-trifluoressigsäurederivate überführt und gaschromatographisch analysiert werden.

\section{Ergebnisse}

Dargestellt werden die Ergebnisse der Úberprüfung der einzelnen Analysenschritte und der des gesamten Analysenganges von Stuhlproben unter Zugabe von Standardlösungen. Anschließend werden Daten hinsichtlich des Gallensäurenmusters nicht-sulfatierter und sulfatierter Gallensäuren im Stuhl beim Menschen mitgeteilt.

\section{Úberprüfung der einzelnen Analysen- schritte}

Zur methodischen Validierung wurden Standardlösungen unterschiedlicher Gallensäuren $(1 \mathrm{mmol} / \mathrm{l})$ verwendet, wobei jeweils $1 \mathrm{ml}$ der entsprechenden Gallensäure in den Versuch eingesetzt wurde. 


\section{Hydrolyse (Tab. 2)}

Bei der Hydrolyse Glycin- und Taurin-konjugierter Gallensäuren treten fast keinerlei Verluste auf, so daß die enzymatische Spaltung als komplett anzusehen ist.

\section{Solvolyse}

Die Ausbeute der Solvolyse, geprüft mit 3a-Sulfolithocholsäure,betrug 79,0 \pm 0,9\% (Mittelwert und dessen Standardabweichung aus 6 Versuchen).

Tab. 2. Wiederfindung bei der Hydrolyse von mit Glycin und Taurin konjugierten Gallensäuren.

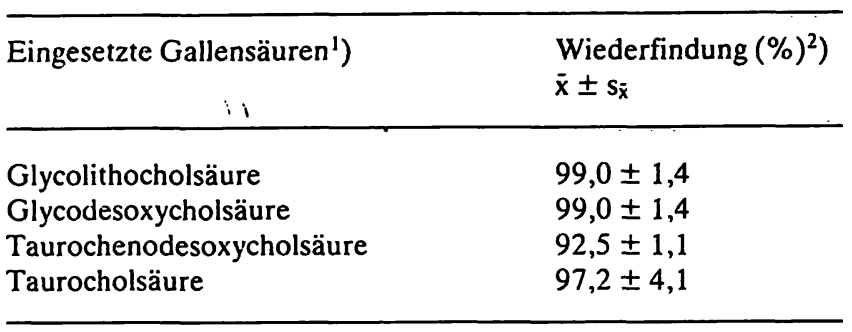

1) Eingesetzt wurde jeweils $1 \mu \mathrm{mol}$ der angegebenen Gallensäuren.

2) Pro Gallensäure wurden 5 Versuche durchgeführt; angegeben sind der Mittelwert $(\bar{x})$ und dessen Standardabweichung $\left(\mathbf{s}_{\overline{\mathbf{x}}}\right)$.

Tab. 3. Wiederfindung von Gallensäuren bei der XAD-2- und Amberlyst-15-Chromatographie.

\begin{tabular}{lll}
\hline Aufgetragene Gallensäuren $^{1}$ ) & $\begin{array}{l}\text { XAD 2 }{ }^{2} \text { ) } \\
\text { Wiederfindung } \\
(\%)\end{array}$ & $\begin{array}{l}\text { Amberlyst } 15^{2} \text { ) } \\
\text { Wiederfindung } \\
(\%)\end{array}$ \\
& $\overline{\mathbf{x}} \pm \mathrm{s}_{\overline{\mathbf{x}}}$ & $\overline{\mathrm{x}} \pm \mathrm{s}_{\overline{\mathbf{x}}}$ \\
\hline
\end{tabular}

\section{Freie Gallensäuren}

Lithocholsäure

Desoxycholsäure

Chenodesoxycholsäure

Ursodesoxycholsäure

Cholsäure

$$
\begin{array}{r}
100,0 \pm 5,0 \\
110,0 \pm 4,5 \\
95,0 \pm 3,1 \\
92,0 \pm 4,5 \\
98,0 \pm 3,6
\end{array}
$$

$93,3 \pm 5,0$

$106,8 \pm 6,5$

$97,6 \pm 3,1$

$107,4 \pm 4,5$

$84,9 \pm 3,6$

Konjugierte Gallensäuren

Glycodesoxycholsäure

Taurocholsäure

$$
93,1 \pm 3,1
$$

$92,6 \pm 2,0$

$89,5 \pm 1,6$

$89,4 \pm 1,3$

Sulfatierte Gallensäuren

$3 \alpha$-Sulfolithocholsäure

$$
91,2 \pm 1,1 \quad 91,0 \pm 1,8
$$

') Aufgetragen wurde jeweils $1 \mu \mathrm{mol}$ der angegebenen Gallensäuren.

$\left.{ }^{2}\right)$ Pro Gallensäure wurden 6 Versuche durchgeführt. Verluste durch gegebenenfalls erforderliche Hydrolyse und Solvolyse sind miteingeschlossen. Angegeben sind der Mittelwert $(\overline{\mathbf{x}})$ und dessen Standardabweichung $\left(s_{\bar{x}}\right)$.
XAD-2- und Amberlyst-15-Chromatographie (Tab. 3)

Zur Ủberprüfung der Wiederfindung wurden unterschiedliche freie, konjugierte und sulfatierte Gallensäuren auf die Säulen aufgebrachţ. Die Verlüste, die durch die vor der gaschromatographischen Analyse erforderlichen Hydrolyse- und Solvolyseschritte verursacht werden, sind in den aufgeführten Ergebnissen mitenthalten.

\section{DEAP-Sephadex LH20-Chromatographie (Abb. 2)}

In diesen Versuchen wurde neben der Wiederfindungsrate für die einzelnen Gallensäuren auch die Trennleistung der Säule überprüft. Deshalb wurden in den Versuchen mit konjugierten Gallensäuren alle Fraktionen der Hydrolyse und bei der Untersuchung der sulfatierten Gallensäuren alle Fraktionen der Solvolyse unterworfen.

\section{Gaschromatographie}

Zur Kontrolle des Meßbereichs der Gaschromatographie wurden 5 Gallensäuren in verschiedenen Konzentrationen untersucht. Als interner Standard wurde 3 $\alpha$-Hydroxy-6-oxo-5 $\alpha$-cholansäure $(1 \mathrm{mmol} / \mathrm{l})$ zugesetzt. Die Standardgerade der einzelnen Gallensäuren wurde über ein ,multilevelCalibration"-Verfahren für eine nichtlineare Responsekurve erstellt. Bei einer Konzentration von $0,025 \mathrm{mmol} / 1$ liegt die relative Standardabweichung bei etwa $5 \%$ und sinkt mit steigender Konzentration (ab 0,5 mmol/l) auf etwa 2\%. Die Abweichungen vom Sollwert liegen bei einer Konzentration von $0,025 \mathrm{mmol} / 1$ bei etwa $25 \%$, betragen jedoch schon ab $0,05 \mathrm{mmol} / 1$ nur noch etwa $5 \%$. Auf Grund unserer Ergebnisse setzten wir als untere Nachweisgrenze mit ausreichender Präzision eine Konzentration von $0,035 \mathrm{mmol} / \mathrm{l}$ fest. Bei mehreren Stuhlanalysen fanden sich häufiger zwei quantitativ bedeutendere Peaks, die wir anhand der in der Literatur (22) angegebenen Daten als $12 \beta$-Hydroxy- und $3 \alpha$, $12 \beta$-Dihydroxy- $5 \alpha$-cholansäuren identifizierten.

Úberprüfung des gesamten Analysenganges fäkaler Gallensäuren (Tab. 4)

Da bei der Natur des Untersuchungsmaterials nicht damit gerechnet werden konnte, daß bei der Analyse fäkaler Gallensäuren die gleichen Wiederfindungsraten erreicht würden wie bẹi den Versuchen mit Standardsubstanzen allein, wurden Wiederfindungsraten und Reproduzierbarkeit anhand von Stuhlpro- 


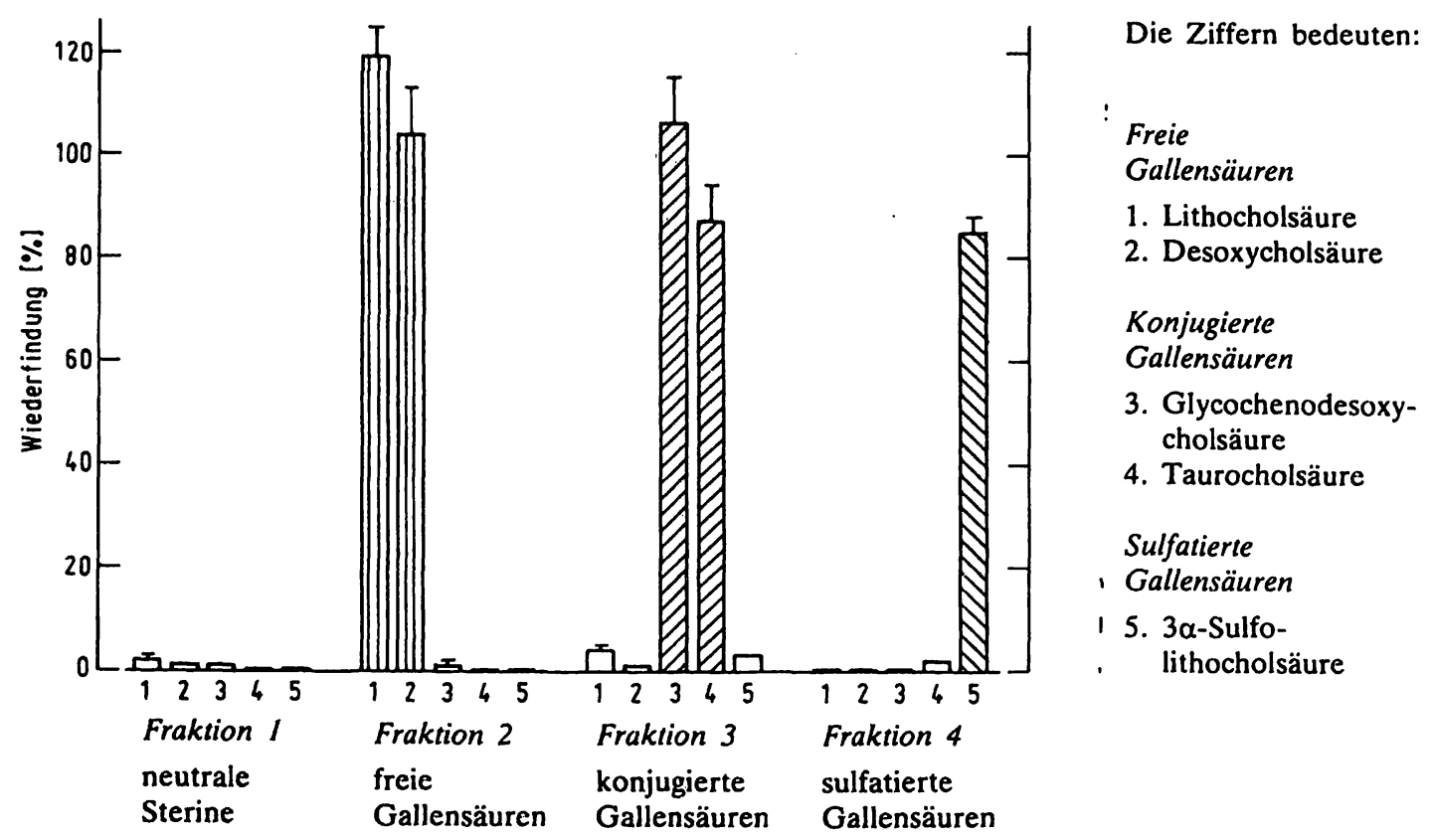

Abb. 2. Trennung und Wiederfindung von Gallensäuren bei der DEAP-Sephadex LH 20-Chromatographie (6 Versuche pro Gallensäure. Angegeben sind Mittelwert und dessen Standardabweichung. Verluste durch Hydrolyse und Solvolyse sind eingeschlossen.)

Tab. 4. Wiederfindung der den Stuhlproben zugesetzten Gallensäuren.

\begin{tabular}{lll}
\hline Eingesetzte Gallensäuren & $\begin{array}{l}\left.\text { Eingesetzte }{ }^{1}\right) \\
\text { Menge }\end{array}$ & $\begin{array}{l}\text { Wiederfindung } \\
\left.(\%)^{2}\right) \\
\overline{\mathbf{x}} \pm s_{\overline{\mathbf{x}}}\end{array}$ \\
\hline
\end{tabular}

Freie Gallensäuren

${ }^{14} \mathrm{C}$-Lithocholsäure

4987 counts/min $\quad 82,8 \pm 4,3$

Konjugierte Gallensäuren ${ }^{3}$ )

Taurocholsäure o. $\quad 1 \mu \mathrm{mol} \quad 93,4+4,1$

Glycodesoxycholsäure

Sulfatierte Gallensäuren

$3 \alpha$-Sulfolithocholsäure

$1 \mu \mathrm{mol}$

$74,6+1,6$

l) Die Gallensäuruen wựden zu 1 g Trockenstuhl zụgesetzt. Pro Gallensäure wurden 6 Versuche durchgeführt.

2) Angegeben sind Mittelwert $(\overline{\mathbf{x}})$ und dessen Standardabweichung $\left(\mathbf{s}_{\overline{\mathbf{x}}}\right)$.

3) In 3 Versuchen wurde Taurocholsäưre, in 3 Versuchen Glycodesoxycholsäure zugesetzt.

ben zusätzlich bestimmt. Dazu wurde bei 6 Patienten nach erfolgter Analyse fäkaler Gallensäuren einem weiteren Aliquot der gefriergetrockneten Stühle vor der Extraktion eine definierte Menge von Gallensäuren ( $1 \mathrm{ml}, 1 \mathrm{mmol} / \mathrm{l}$ ) zugesetzt, die auf Grund der vorhergehenden Analyse nicht in der Stuhlprobe vorhanden waren, und auf ihre Wiederfindung hin geprüft. Lediglich für Lithocholsäure ließ sich naturgemäß keine Stuhlprobe finden, die frei von dieser Gallensäure war, weshalb wir ${ }^{14} \mathrm{C}$-markierte Lithocholsäure für die Wiederfindungsuntersuchungen benutzten. Aus den Wiederfindungsraten der zugesetzten Gallensäuren wurde für jede Fraktion (freic, konjugierte, sulfatierte Gallensäuren) ein Faktor errechnet, mit dem die bei späteren Analysen auftretenden, für die einzelnen Fraktionen unterschiedlichen Verluste korrigiert wurden.

\section{Ausscheidungsmuster nicht-sulfatierter und sulfatierter Gallensäuren im Stuhl von Referenzpersonen}

Die unter Anwendung der beschriebenen Methode erzielten Ergebnisse sind in den Tabellen 5 und 6 zusammengefaßt. Den größten Anteil $(89,6 \%)$ der fäkalen Gallensäuren stellen die sekundären Gallensäuren dar; weiterhin sind fast alle Gallensäuren $(92,9 \%)$ im Stuhl nicht mehr glycin- oder taurinkonjugiert. Die sulfatierten Gallensäuren stellen mit $4,4 \%$ nur einen kleinen Anteil an der Gesamtausscheidung dar. Ein signifikanter Unterschied im Sulfatierungsgrad der einzelnen Gallensäuren liegt nicht vor. 


\section{Diskussion}

\section{Methodik}

Der wesentliche Vorteil der von uns beschriebenen Methode liegt in der voneinander unabhängigen $\mathrm{Be}-$ stimmung der nicht-sulfatierten und sulfatierten Gallensäuren, die durch die säulenchromatographische Trennung auf DEAP-Sephadex LH20 ermög-

Tab. 5. Ausscheidungsmuster fäkaler Gallensäuren bei Referenzpersonen $(n=15)$.

\begin{tabular}{|c|c|c|}
\hline & $\begin{array}{l}\text { Konzentra- } \\
\left.\text { tion' }{ }^{1}\right) \\
\text { (mg/g Trok- } \\
\text { kenstuhl) }\end{array}$ & $\begin{array}{l}\text { Anteil } \\
\left.(\%) \text { an }^{2}\right) \\
\text { Gesamt- } \\
\text { ausschei- } \\
\text { dung }\end{array}$ \\
\hline & $\bar{x} \pm s_{\bar{x}}$ & $\overline{\mathbf{x}} \pm s_{\bar{x}}$ \\
\hline Gesamtgallensäuren & $3,85 \pm 0,63$ & 100,0 \\
\hline Primäre Gallensäuren & $0,36 \pm 0,13$ & $10,4 \pm 2,6$ \\
\hline $\begin{array}{l}\text { Chenodesoxycholsäure } \\
\text { Cholsäure }\end{array}$ & $\begin{array}{l}0,19 \pm 0,06 \\
0,17 \pm 0,07\end{array}$ & $\begin{array}{l}6,1 \pm 1,4 \\
4,3 \pm 1,3\end{array}$ \\
\hline Sekundäre Gallensäuren & $3,49 \pm 0,61$ & $89,6 \pm 2,6$ \\
\hline $\begin{array}{l}\text { Lithocholsäure } \\
\text { Desoxycholsäure } \\
\text { Ursodesoxycholsäure } \\
3 \alpha, 12 \beta \text {-Dihydroxy-5 } \alpha \text {-cholansäure } \\
12 \beta \text {-Hydroxy-5 } \alpha \text {-cholansäure }\end{array}$ & $\begin{array}{l}1,54 \pm 0,41 \\
1,62 \pm 0,37 \\
0,16 \pm 0,10 \\
0,12 \pm 0,04 \\
0,06 \pm 0,01\end{array}$ & $\begin{array}{r}43,6 \pm 3,8 \\
37,6 \pm 3,3 \\
4,2 \pm 2,0 \\
2,5 \pm 0,8 \\
1,7 \pm 0,4\end{array}$ \\
\hline Nicht-sulfatierte Gallensäuren & $3,67 \pm 0,59$ & $95,6 \pm 1,2$ \\
\hline $\begin{array}{l}\text { frei } \\
\text { konjugiert }\end{array}$ & $\begin{array}{l}3,58 \pm 0,58 \\
0,10 \pm 0,03\end{array}$ & $\begin{array}{r}92,9 \pm 1,9 \\
2,7 \pm 0,7\end{array}$ \\
\hline Sulfatierte Gallensäuren & $0,18 \pm 0,06$ & $4,4 \pm 1,2$ \\
\hline
\end{tabular}

1) Zur Berechnung wurde jeweils das Molekulargewicht der entsprechenden freien Gallensäure eingesetzt.

$\left.{ }^{2}\right)$ Die Berechnung des Anteils erfolgte über die molare Konzentration der einzelnen Gallensäuren. licht wird. Demgegenüber besteht eine größere Fehlermöglichkeit, wenn man den Anteil der Sulfatester indirekt bestimmt, imdem man die Differenz von zwei Analysen (mit und ohne Solvolyseschritt) bildet.

Durch das sofortige Einfrieren der gesammelten Stuhlproben wird ein bakterieller Abbau der Gallensäuren verhindert. Die mechanische Homogenisierung des Lyophilisates gewährleistet eine Homogenität der Untersuchungsprobe.

Bei der Extraktion der Gallensäuren aus dem Stuhl ist zu berücksichtigen, daß ein beträchtlicher Teil der Gallensäuren an feste Stuhlbestandteile wie Bakterien (23) und Faserstoffe, wie Lignin (24), gebunden ist. Die drei von uns verwendeten Lösungsmittel unterschiedlicher Polarität (25) führten nicht nur auch in unserer Hand zu guten Wiederfindungsraten der dem Stuhl (exogen) zugesetzten Gallensäuren, sondern auch zu guten Extraktionsergebnissen von endogenen, d.h. dem Patienten verabreichten (radioaktiv markierten) und somit schon im Stuhl vorhandenen Gallensäuren (25). Die XAD-2-Chromatographie wird zur Abtrennung von Verunreinigungen angewendet, insbesondere von Salzen, die die anschließende Chromatographie an Amberlyst-15 stören würden. Dieser Kationenaustauscher dient zur Úberführung der Gallensäuren in ihre protonierte Form als Voraussetzung für die sich daran anschlieBende Chromatographie an DEAP-Sephadex LH2O. Hierbei handelt es sich um ein lipophiles Gel zur Auftrennung von in organischen Lösungsmitteln gelösten Gallensäuren, die hauptsächlich nach dem Prinzip der Ionenaustauschchromatographie aufgetrennt werden. Da der Anteil der konjugierten Gallensäuren an der Gesamtausscheidung der fäkalen Gallensäuren mit weniger als $3 \%$ recht klein ist, ermöglicht die von uns geübte gemeinsame Elution der

Tab. 6. Verteilung der einzelnen Gallensäuren auf die Fraktion der freien, konjugierten und sulfatierten Gallensäuren bei Referenzpersonen $(n=15)$ (angegeben in Prozent der Gesamtausscheidung, molaritätsbezogen).

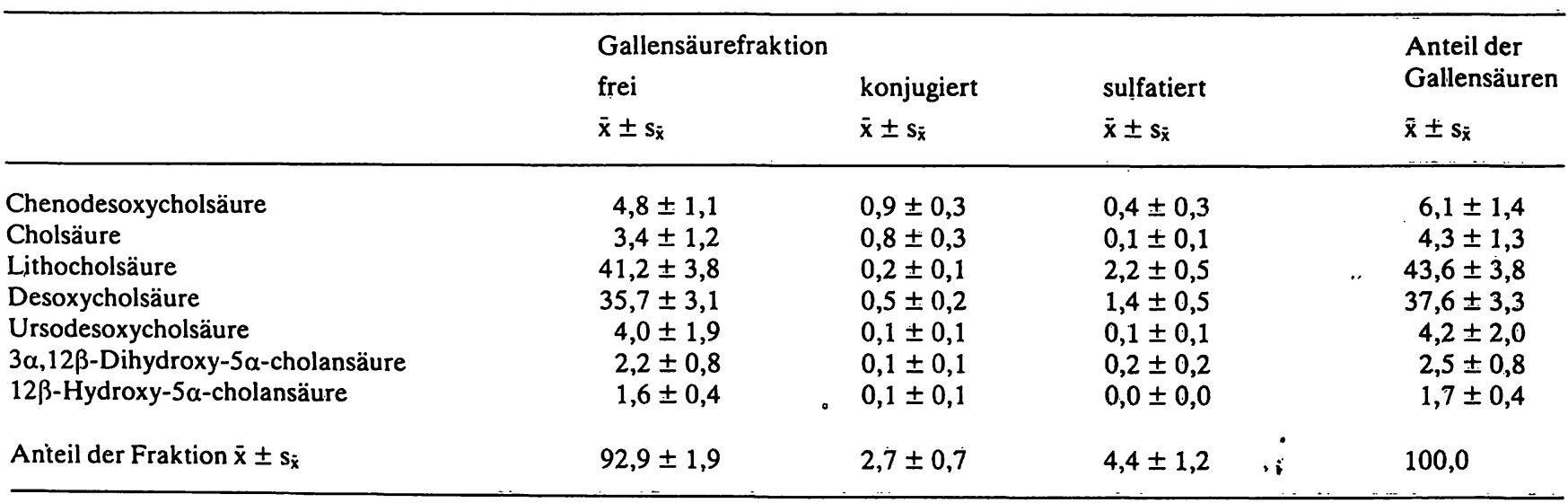


Glycin- und Taurin-Konjugate eine genauere Quantifịzierung. Wie unsere Ergebnisse belegen, zeichneten sich XAD-2- und Amberlyst-15-Chromatographie durch eine gute Reproduzierbarkeit und geringe Verluste aus. Bei der Úberprüfung der Trenneigenschaften von DEAP-Sephadex LH20 war der Ubertritt der konjugierten Gallensäuren in die Sulfatesterfraktion mit $2,5 \%$ äußerst gering, so daß fälschlich hohe Werte für die sulfatierten Gallensäuren wegen des geringen Anteils der Gallensäurenkonjugate an der Gesamtausscheidung praktisch auszuschließen sind. Da auch kein wesentlicher Ubertritt der sulfatierten Gallensäuren in die vorausgehenden Fraktionen nachzuweisen war, ist der Fehler einer falsch niedrigen Messung der Sulfatesterfraktion als gering anzusehen. Um eine gute Trennung zu erzielen, sollte unseren Erfahrungen zufolge die Durchflußgeschwindigkeit nicht kleiner als 20 bis $22 \mathrm{ml} /$ Stunde betragen.

Auf Grund des gewählten Extraktionsverfahrens mit Sep Pak ${ }^{\mathrm{TM}}-\mathrm{C}_{18}$ Kartuschen war die Durchführung der enzymatischen Hydrolyse fast verlustfrei.

Das von uns gewählte Solvolyseverfahren mit etherischer Salzsäure ermöglicht eine sofortige Weiterverarbeitung der Probe nach Abblasen des Solvolysemittels; eine Extraktion der freigesetzten Gallensäuren ist somit nicht erforderlich.

Zur gaschromatographischen Analyse wurden verschiedene Derivate (Methylester, Methylester-trimethylsilylether, Methylester-heptafluorobutyrat, Methylester-acetat) und Säulenmaterialien (Poly S 176 und SP 2250) getestet, die optimale Trennung erzielten wir jedoch mit Methylester-trifluoroacetatDerivaten auf einer 1\% QF1 Säule.

\section{Gallensäureausscheidung bei Referenzper- sonen}

Für die Gesamtmenge der im Stuhl ausgeschiedenen Gallensäuren werden sehr unterschiedliche Angaben gemacht. Obgleich Menge und Zusammensetzung der Gallensäureausscheidung sicherlich auch individuellen Schwankungen infolge ernährungsund stoffwechselbedingter Faktoren unterworfen sein dürfte, ist die Unterschiedlichkeit der Angaben auch auf die Anwendung verschiedener Bestimmungsverfahren zurückzuführen. Hierauf weist eine vergleichende Gegenüberstellung der Daten (26) hin. Diese Unterschiede betreffen sowohl die Angaben der Gesamtausscheidung pro Zeiteinheit als auch die Gallensäuremenge pro Gewichtseinheit Stuhl. Während Untersuchungen an amerikanischen Referenzpersonen eine Ausscheidung von $10,0 \mathrm{mg} / \mathrm{g}$
Trockenstuhl ergaben (27), wurde an einem englischen Untersuchungsgut ein mittlerer Wert von 4,7 $\mathrm{mg} / \mathrm{g}$ (28) ermittelt, vergleichbar mit unseren Ergebnissen. Der Eindruck, daß die durch chemische Analysen des Stuhls ermittelten Werte für die Gallensäureausscheidung generell niedriger liegen als die durch Isotopenmethoden (29) erzielten Ergebnisse, wurde durch die simultane Anwendung beider Verfahren bestätigt (15). Durch Einführung eines Solvolyse-Schrittes (30) in eine etablierte chemische Bestimmungsmethode (26) wurden jedoch höhere Ausscheidungswerte ermittelt, die den durch Isotopentechniken erzielten Ergebnissen entsprachen. Vernachlässigte man den durch Differenzbildung ermittelten Anteil der sulfatierten Gallensäuren, der etwa 1/3 der Gesamtausscheidung ausmachte, so waren die Ergebnisse vergleichbar mit den Ergebnissen anderer chemischer Analyseverfahren. Demgegenüber konnten andere Autoren durch eine zusätzliche Solvolyse, also ohne eine chromatographische Abtrennung durchzuführen, nur Spuren (15) bzw. keine signifikànten Mengen (18) nachweisen.

Eine chromatographische Abtrennung der sulfatierten Gallensäuren von den nicht-sulfatierten mittels Sephadex-LH20 ergab einen Anteil der Sulfatester von etwa $12 \%$ (17), während mit gleicher Technik andernorts (31) keine sulfatierten Gallensäuren im Stuhl nachgewiesen werden konnten. Da der Gebrauch dieses Gels neben anderen Trennproblemen insbesondere leicht eine Kontamination der Sulfatfraktion durch die (in Stuhlproben) in größerer Menge vorhandenen nicht-sulfatierten Gallensäuren erfolgen kann (32), wählten wir zur genaueren Analyse die DEAP-Sephadex LH20-Säule, die eine bessere Trennleistung aufweist (19). Mit dieser Methode bestimmten wir den Anteil der sulfatierten Gallensäuren im Stuhl mit 4,4\%, wobei aufgrund unserer Ergebnisse kein Unterschied im Sulfatierungsgrad der einzelnen Gallensäuren vorzuliegen scheint. Erwähnenswert erscheint uns, daß kürzlich, ebenfalls unter Verwendung des DEAP-Sephadex LH20 Gels, der Anteil der sulfatierten Gallensäuren an der Gallensäure-Ausscheidung aus dem Ileum (über ein Ileostoma) mit 1,5\% ermittelt wurde (33).

Die weitere Analyse des Gallensäureausscheidungsmusters entsprach den bekannten Angaben in der Literatur. Erwartungsgemäß $(18,34)$ waren Glycinund Taurin-Konjugate praktisch vollständig (92,9\%) durch die Einwirkung der Dickdarmbakterien gespalten worden.

Ebenfalls in Ubereinstimmung mit früheren Untersuchungen $(18,27,28,35,36)$ lag nur ein geringer Anteil als primäre Gallensäuren Chenodesoxychol- 
säure und Cholsäure vor; der überwiegende Teil $(89,6 \%)$ war durch bakterielle Dehydroxylierung in sekundäre Gallensäuren überführt worden.

\section{Klinische Bedeutung}

Mehrere Untersuchungen haben eine Abhängigkeit der biologischen Wirkung der Gallensäuren von ihrer Struktur gezeigt, was in mehrerer Hinsicht auch für die unterschiedlichen Eigenschaften nichtsulfatierter und sulfatierter Gallensäuren gilt. So wird zum Beispiel die stark hepatotoxische Monohydroxygallensäure Lithocholsäure (37) bei Cholestase vermehrt sulfatiert (3-6), was zu einer vermehrten Ausscheidung über Niere und Darm führt. Darüber hinaus weist der Lithocholsäuresulfatester aber auch eine deutlich geringere Toxizität auf (7, 38). Úber diesen Mechaniśmus wird auch im Rahmen der medikamentösen Gallensteinauflösung mit Chenodesoxỳcholsäure die vermehrt anfallende Lithocholsäure metabolisiert (39). Bei Tieren mit geringer Kapazität zur Sulfatierung kommt es hingegen zu einer Akkumulation von Lithocholsäure, die morphologische und funktionelle Veränderungen der Leber bewirkt (8). Neuere Untersuchungen (40, 41) zeigen jedoch, daß die Sulfatierung nicht generell die Entstehung einer intrahepatischen Cholestase verhindern kann. Ein weiteres Beispiel für die Struktur-Wirkungs-Beziehung bei Gallensäuren ist die Hemmung des Wasser- und Elektrolyttransports im Colon (42), die sich nur für Dihydroxygallensäuren nachweisen läßt, die ihre Hydroxylgruppen in $\alpha$-Position am Steroidgerüst tragen $(43,44)$. Dieser sekretorische Effekt, der sich klinisch bei Erkrankungen oder Resektionen des terminalen Ileums infolge einer Gallensäurenmalabsorption zeigt (45), wird jedoch eigenen Untersuchungen zufolge (9) bei sulfatierten Dihydroxygallensäuren nicht gefunden. Schließlich sprechen auch einige Untersuchungen, die sich mit der Co-Carcinogenität von Gallensäuren $(46,47)$ bei der Entstehung des Coloncarcinoms be- fassen, für eine unterschiedliche Wirkung verschieden strukturierter Gallensäuren. Für èine besondere Rolle der sekundären Gallensäuren hierbei sprechen nicht nur tierexperimentelle Untersuchungen (48), sondern auch die Beobachtung, daß sich nur die sekundären Gallensäuren, nicht ab́er die primären, im AMES-Test als co-mutagen erwiessen (49). Darüber hinaus konnte nur mit nicht-sulfatierter Lithocholsäure eine Transformiërung von Embryozellen des Syrischen Hamsters ausgelöst werden, nicht jedoch mit dem entsprechenden Sulfatester (50). Ob die Sulfatierung sekundärer Gallensäuren deren comutagene Wirkung aufhebt, wird zur Zeit von uns untersucht.

Die aufgeführten Beispiele belegen eine geringere Toxizität der sulfatierten Gallensäuren, deren Anteil an der Gesamtausscheidung durch die hepatische Sulfatierung $(14,51,52)$ einerseits und die bakterielle Desulfatierung im Darm (12) andererseits bestimmt wird. Augenblicklich gibt es noch keinen Weg, die Aktivität der hepatischen Sulfotransferase $(53,54)$, deren Aktivität jedoch individuelle Unterschiede aufweist (55), zu beeinflussen. In der nahen Zukunft jedoch erscheint es uns geboten, mittels der fäkalen Gallensäureanalyse sowohl die Rolle der sulfatierten Gallensäuren bei Erkrankungen des Colons, insbesondere beim Coloncarcinom, als auch die mögliche Beeinflussung des Sulfatierungsgrades durch Veränderungen der bakteriellen Mikroflora im Dickdarm zu erforschen. Untersuchungen in diese Richtung sind von uns begonnen worden.

\section{Danksagung}

Das Projekt wurde seitens der Deutschen Forschungsgemeinschaft (SFB 102, Projekt D2) gefördert.

R. Tandon war Stipendiat der Alexander von Humboldt-Stiftung; jetzige Adresse: All India Institute of Medical Sciences, Dept. of Gastroenterology, New Delhi, India.

Den technischen Assistentinnen A. Scheel und C. Wirth danken wir für ihre zuverlässige und sorgfältige Mitarbeit.

\section{Literatur}

1. Small, D. M., Dowling, R. H. \& Redinger, R. N. (1972) Arch. Intern Med. 130, 552-573.

2. Palmer, R. H. (1967) Proc. Nat. Acad. Sci. USA 58, 10471050.

3. Stiehl, A. (1972) In: Bile Acids in Human Diseases (Back, $P$. \& Gerok, W., Hrsg.), pp. 73-77, F. K. Schattauer Verlag, Stuttgart-New York.

4. Back, R. (1973) Clin. Chim. Acta 4, 199-207.

5. Makino, I., Hashimoto, H., Shinozaki, K., Yoshino, K. \& Nakagawa, S. (1975) Gastroenterology 68, 545-553.

6. van Berge Henegouwen, G. P., Brandt, K.-H., Eyssen, H. \& Parmentier, G. (1976) Gut 17, 861-869.

7. Czygan, P. \& Stiehl, A. (1975) Z. Gastroenterol. 13, 468473.

8. Gadacz, T. R., Allan, R. N., Mark, E. \& Hofmann, A. F. (1976) Gastroenterology 70, 1125-1129.

9. Breuer, N. F., Rampton, D. S., Tammar, A., Murphy, G. M. \& Dowling, R. H. (1983) Gastroenterology 84, 969-977.

10. Stiehl, A., Thaler, M. M. \& Admirand, W. H. (1972) N. Engl. J. Med. 286, 858-861.

11. Low Beer, T. S., Tyor, M. P. \& Lack, L. (1969) Gastroenterology 56, 721-726.

12. Cowen, A. E., Kormann, M. G., Hpfmann, A. F., Cass, O. W. \& Coffin, S. B. (1975) Gastroenterology 69, 67-76. 
13. De Witt, E. H. \& Lack, L. (1980) Amer. J. Physiol. 238, G34-39.

14. Palmer, R. H. (1971) J. Lipid Res. 12, 680-687.

15. Subbiah, M. T. R., Tyler, N. E., Buscaglia, M. D. \& Marai, L. (1976) J. Lipid Res. 17, 78-84.

16. Podesta, M. T., Murphy, G. M., Sladen, G. E. \& Dowling, R. H. (1978) Clin. Sci. Mol. Med. 54, 32 p.

17. Salvioli, G. \& Salati, R. (1979) Gut 20, 698-704.

18. Mc Junkin, B., Fromm, H., Sarva, R. P. \& Amin, P. (1981) Gastroenterology 30, 1454-1464.

19. Alme, B., Bremmelgaard, A., Sjövall, J. \& Thomassen, P. (1977) J. Lipid Res. 18, 339-362.

20. Roseleur, O. J. \& van Gent, C. M. (1976) Clin. Chim. Acta 66, 269-272.

21. van Berge-Henegouwen, G. P., Allan, R. N., Hofmann, A. F. \& Yu, P. Y. S. (1977) J. Lipid Res. 18, 118-122.

22. Sjövall, J. (1962) Acta Chem. Scand. 16, 1761-1764.

23. Gustafsson, B. E. \& Norman, A. (1962) Proc. Soc. Exp. Biol. Med. 110, 387-389.

24. Eastwood, M. A. \& Hamilton, D. (1968) Biochim. Biophys. Acta 152, 165-173.

25. Setchell, K. D. R., Lawson, A. M., Sjövall, J. \& Tanida, N. (1980) VI. International Bile Acid Meeting, Bile Acids and Lipids, Freiburg/Br, Abstract book p. 71.

26. Grundy, S. M., Ahrens, E. H. \& Miettinen, T. A. (1965) J. Lipid Res. 6, 397-410.

27. Reddy, B. S. \& Wynder, E. L. (1977) Cancer 39, 25332539.

28. Hill, M. J., Drasar, B. S., Williams, R. E. O., Meader, T. W., Cox, A. G., Simpson, J. E. P. \& Morson, B. C. (1975) Lancet I, 535-539.

29. Lindstedt, S. (1957) Acta Physiol. Scand. 40, 1-9.

30. Podesta, M. T., Murphy, G. M. \& Dowling, R. H. (1980) J. Chromatogr. 182, 293-300.

31. Islam, M. A., Raicht, R. F. \& Cohen, B. I. (1981) Anal. Biochem. 112, 371-377.

32. Eyssen, H. J., Parmentier, G. G. \& Mertens, J. A. (1976) Eur. J. Biochem. 66, 507-514.

33. Raedsch, R., Stiehl, A., Walker, S., Czygan, P. \& Kommerell, B. (1983) Z. Gastroenterol. 21, 426.

34. Danielsson, H. (1963) In: Advances in Lipid Research (Paoletti, R. \& Kritchevsky, D., Hrsg.), Bd. 1, 335-385, Academic Press; New York-London.
35. Hofmann, A. F. (1972) Arch. Intern. Med. 130, 597-604.

36. Aldini, R., Roda, A., Festi, D., Sama, C., Mazella, G., Bazzoli, F., Morselli, A. M., Roda, E. \& Barbara, L. (1982) Dig. Dis. Sci. 27, 495-502.

37. Palmer, R. H. (1972) Arch. Intern. Med. 130, 606-617.

38. Leuschner, U., Czygan, P. \& Stiehl, A. (1975) Verh. Dtsch. Ges. Inn. Med. 81, 1311-1313.

39. Allan, R. N., Thistle, J. L., Hofmann, A. F., Carter, J. A. \& Yu, P. Y. S. (1976) Gut 17, 405-412.

40. Yousef, I. M., Tuchweber, B., Vonk, R. J., Masse, D., Audet, M. \& Roy, C. C. (1981) Gastroenterology 80, 233-241.

41. Mathis, U., Karlaganis, G. \& Preisig, R. (1983) Gastroenterology $85,674-681$.

42. Mekhjian, H. S., Phillips, S. F. \& Hofmann, A. F. (1971) J. Clin. Invest. 50, 1569-1577.

43. Chadwick, V. S., Gaginella, T. S., Carlson, G. L., Debongnie, J. C., Phillips, S. F. \& Hofmann, A. F. (1979) J. Lab. Clin. Med. 94, 661-674.

44. Gordon, S. J., Kinsey, M. D., Magen, J. S., Joseph, R. E. \& Kowlessar, O. D. (1979) Gastroenterology 77, 38-44.

45. Hofmann, A. (1967) Gastroenterology 52, 752-757.

46. Hill, M. J. (1976) In: The Bile Acids (Nair, P. P. \& Kritchevsky, D., Hrsg.), Bd. 3 (Metabolism), 169-200, Plenum Press, New York.

47. Nigro, N. D. \& Campbell, R. L. (1976) In: The Bile Acids (Nair, P. P. \& Kritchevsky, D., Hrsg.), Bd. 3 (Metabolism), 155-168, Plenum Press, New York.

48. Reddy, B. S. \& Watanabe, K. (1979) Cancer Res. 39, 15211524.

49. Wilpart, M., Mainguet, P., Maskens, A. \& Roberfroid, M (1983) Carcinogenesis 4, 45-48.

50. Kelsey, M. I. \& Pienta, R. J. (1979) Cancer Lett. 9, 143149.

51. Liersch, M. \& Stiehl, A. (1974) Z. Gastroenterol. 12, 131134.

52. Cowen, A. E., Korman, M. G., Hofmann, A. F. \& Cass, O. W. (1975) Gastroenterology 69, 59-66.

53. Chen, L. J., Bolt, R. J. \& Admirand, W. H. (1977) Biochim. Biophys. Acta 480, 219-227.

54. Hammermann, K. J., Chen, L. J., Corugedo, A. F. \& Earnest, D. L. (1978) Gastroenterology 75, 1021-1025.

55. Marks, J. W., Sue, S. O., Pearlman, B. J., Bonorris, G. G., Varady, P., Lachin, J. M. \& Schoenfield, L. J. (1981) J. Clin. Invest. $68,1190-1196$.

Dr. med. Norbert Breuer

Medizinische Klinik und Poliklinik

Abteilung für Gastroenterologie

Universitätsklinikum der Gesamthochschule Essen

Hufelandstraße 55

D-4300 Essen 1 